\title{
Treatment of breast cancer based on FOLFOX4 therapy for colon cancer: A case report.
}

\author{
Jinyin Yan, Haifeng Cai*, Jinghua Zhang, Bing Zhang, Ning Li, Wanning Hu \\ Department of Breast Surgery, Tangshan People's Hospital, 65 Sheng-li Road, Hebei, 063000, PR China
}

\begin{abstract}
In the United States, breast cancer is the commonly occurring cancer among women is leading second place related death next to lung cancer. Person having breast cancer also faces health issues like genetic, socio-demographic, and lifestyle features and in addition health challenges of the potential for recurrence or development of a second primary cancer. In treatment of colon cancer, the drug named as Fluoropyrimidines plays a vital role because it provides safe treatment for patients having this disease. In recent days, there is a development in the treatment of breast cancer at the early stages. Fluorouracil, Leucovorin, and Oxaliplatin (FOLFOX) are used for chemotherapy regimens selection by genetic methods. A genetic model is used to evaluate chemotherapy sensitivity by examining gene expression and predictive capacity.
\end{abstract}

Keywords: Brest cancer, FOLFOX 4, Colon cancer.

Accepted on May 23, 2017

\section{Introduction}

Breast cancer remains the most common cancer among the women, with more than 230,000 new diagnoses and 40,000 deaths each year in USA, along with decrements in quality of life. Increased clinical genomic profiling of breast tumors, in combination with traditional factors such as age, tumor size, and grade, determine recurrence estimates and guide adjuvant treatment decisions. A key challenge for physicians and their patients in the management of breast cancer in making decisions about the treatment options relates to the use of adjuvant chemotherapy, following surgical resection of the tumor. Adjuvant chemotherapy helps to detect the tumor at early stage but it is suitable for only limited number of patients. The effectiveness of these interventions is based on risk stratification of patients and has involved the use of validated computer-based models using algorithms designed to calculate the rates for recurrence of the disease and neoadjuvant treatment may reduce the risk of recurrence. Neoadjuvant treatment is widely used to treat cancer, especially for patients with advanced stage cancer. Neoadjuvant treatment is also increasingly applied to patients with initially operable breast cancer (BC). Preoperative reduction of tumor volume can lead to better resection results and prognosis. However, not all patients can benefit from neoadjuvant therapy. A pathologically complete response (pCR) is predicted in less than $30 \%$ of patients who undergo neoadjuvant therapy [1]. In addition, treatment outcomes tend to be better with individualized management [2]. Therefore, a more individualized approach for neoadjuvant treatment is urgently needed. Here we describe a case in which we tried to change the chemotherapy regimen after genetic testing, instead of using the regimen recommended in the National Comprehensive Cancer Network (NCCN) guide.

SIRFLOX was considered to access usefulness and care in addition of Selective Internal Radiation Therapy (SIRT) with yttrium-90 resin microspheres to standard Fluorouracil, Leucovorin, and Oxaliplatin (FOLFOX) established chemotherapy on patients with untreated metastatic colorectal cancer already. Chemotherapy-naïve patients using liver metastases plus or minus limited extrahepatic metastases were allotted in random way to obtain either modified FOLFOX (mFOLFOX6; control) or mFOLFOX6 plus SIRT (SIRT) plus or minus bevacizumab. The main argument concluded was Progression-Free Survival (PFS) as obtained by independent centralized radiology examination blinded to study arm [3]. Randomized, open-label, multicenter, phase III trial, patients has 18-24 weeks of induction chemotherapy with XELOX or FOLFOX and attained disease control in random assignment at center (1:1) to obtain care therapy of capecitabine or only observation till disease development. The main conclusion was the PFS from randomization and next is Overall Survival (OS), PFS from induction treatment (PFS2) and safety. This analysis was performed to treat [4]. Patient who has the metastatic Colorectal Cancer (mCRC) were randomized to accept firstline therapy with mFOLFOX6 plus aflibercept $(4 \mathrm{mg} / \mathrm{kg})$ or mFOLFOX6 only. The phase II concludes by stating PFS rate at 12 months in every arm and study between the arms was a pre-planned secondary examination [5]. 


\section{Case Report}

We present the case of a 29-year-old woman who has no family history of $\mathrm{BC}$ and colorectal cancer. She was admitted to the hospital on 26 April 2012 after detection of a mass located in her left breast by self-palpation. She was diagnosed with BC after breast biopsy (pT3NxcM0) (Figure 1). Immunohistochemistry showed the presence of HER2 (3+) (human epidermal growth factor receptor 2), ER (-), PR (-) and Ki-67 (+20\%).
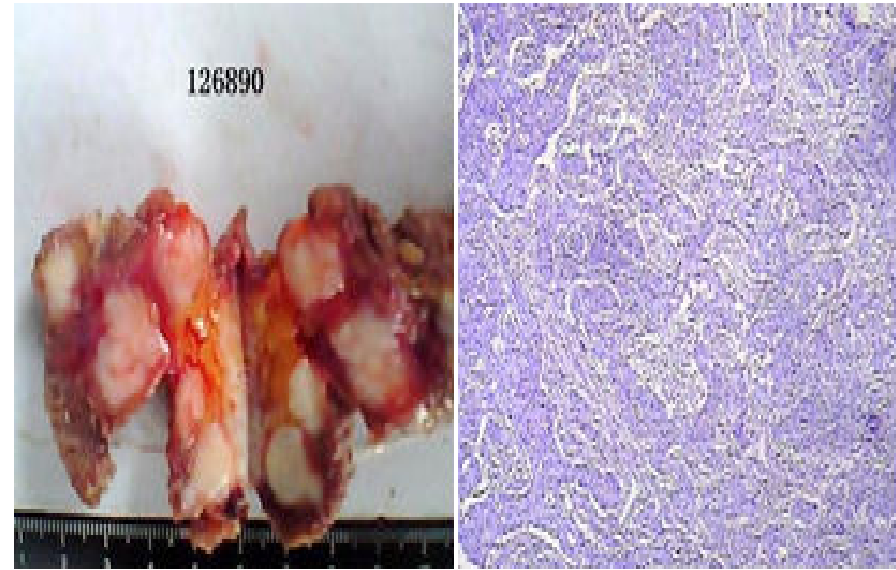

Figure 1. Outline and histological findings of breast biopsy (HE staining, 400X).

Subsequently, she received chemotherapy with paclitaxel and herceptin each week for 12 weeks. The following histological findings were obtained on 9 August 2012 after modified radical mastectomy (left): infiltrating ductal cancer (PT3N3Mx) with microscopically cancer of the breast; a score of G1 according to the Miller-Payne Grading System; and $14 / 61$ axillary lymph nodes. The chemotherapy plan was modified, and docetaxel and epirubicin were administered each week for 12 weeks. Breast ultrasound results showed a hypoechoic nodule (lymphadenopathy) in the left pectoralis major muscle is represented in Figure 2a.

Immunohistochemistry showed the presence of HER2 $(2+)$, ER (-), PR (-) and Ki-67 (+30\%). A breast biopsy, which showed that the metastatic adenocarcinoma was of breast origin, was performed on 26 September 2012. Immunohistochemistry showed the presence of HER2 (2+), ER (-), PR (-) and Ki-67 $(+30 \%)$. The patient subsequently underwent genetic testing (Beijing ACCB Biotech Ltd' Biomark XP), which showed low excision repair cross-complementing gene 1 (ERCC1) and low thymidylate synthase (TYMS). The patient received chemotherapy with FOLFOX4 each week for 12 weeks. The pectoralis and pectoralis minor muscles were excised on 9 May 2013. Histological findings showed the absence of tumor cells. Fat necrosis was observed in the fat and fibrous tissues at the tip of the axilla along with fat vacuoles and inflammatory cell infiltration. Subclavian lymph node hyperplasia was reactivated. A pCR was achieved is shown in Figures $2 b$ and 2c. As of this writing, the patient is in a satisfactory condition (2014-8-6).

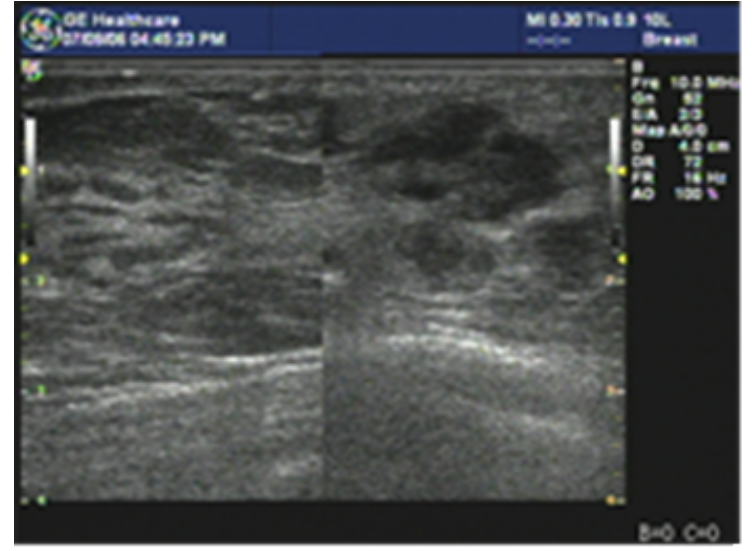

A

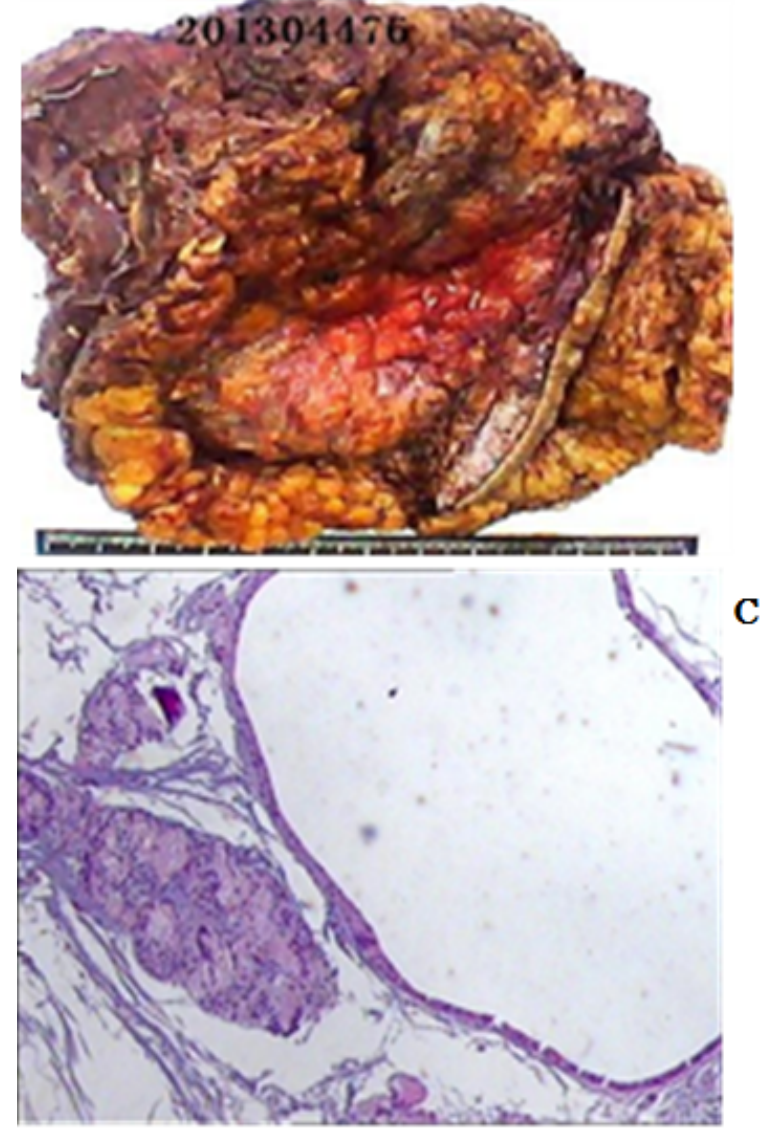

B

$\mathbf{C}$

Figure 2. Ultrasound of the left breast after modified radical mastectomy and outline and histological findings of the pectoralis and pectoralis minor muscles (HE staining, 100X).

\section{Discussion}

Patient with stage II and III cancer is treated by adjuvant therapy with 5-Fluorouracil (5FU) where this method is recommended before 35 years ago by stating the welfares on adjuvant treatment with fluoropyrimidine after surgery. The advantages of adjuvant systemic fluoropyrimidine based treatment was attained by the randomized data after resection of stage Dukes' B2 or C colon carcinoma, was derivated from the Intergroup (INT) 0035 trial. For stage II disease, this trial recognized for 12 months of 5FU and levamisol (lev) as standard of care. Lev role is challenged and substituted by 
Leucovorin (LV) in grouping with 5FU. The duration of treatment is reduced to 6-8 months and so $5 \mathrm{FU}$ and $\mathrm{LV}$ directed either as bolus like Roswell Park or Mayo Clinic regimen or as bolus and continuous infusion (LV5FU2) appeared as standard adjuvant treatment for colon cancer [6]. For adjuvant therapy, the decision making method is stated as a time intensive dialogue among the patient and her oncologist. Most commonly an adjuvant hormonal therapy and chemotherapy are arranged to reduce the risk for relapse by removing microscopic deposits of malignancy because this have not been removed by primary therapy like urgery and radiation therapy. With a particular patient, the value of adjuvant therapy is changeable.

To treat particular patient, many tools are utilized. The neoadjuvant approach can be used to downstage the disease, potentially reduce the extent of surgery, and test the efficacy of therapy administered to patients for individualization of treatment. Biomarkers document observes the neoadjuvant chemotherapy (NAC)'s efficiency to treat and save patients from toxicity affected by non-effective drugs [7]. Further improvements in the treatment of $\mathrm{BC}$ can be expected with better understanding of $\mathrm{BC}$ pathophysiology and with biologically oriented therapeutic interventions. Moreover, patients who are likely to take advantage of specific therapies can be identified. Prognostic data is given by many tools about risk for decline in a specific patient and predict the advantages of adjuvant systemic therapy. So that the dissimilarity among the population-based examination and genomic-based information are created that confuses the decision-making about adjuvant therapy. All measured tests can satisfactorily predict the presence of disease at final pathology, but none of them are able to reliably predict a pCR [8]. For new therapies, preoperative trials can prevent adaptive resistance and pretreatments that could induce complications in an advanced disease setting. The American Joint Committee on Cancer response criteria for $\mathrm{BC}$ is clinically useful in evaluating NAC response but information on FOLFOX4 is not available in the NCCN guide [9]. Differences in gene expression might explain variations in treatment outcomes.

Nearly 4 to 5 percentage of patients suffered from breast cancer is benefited by the adjuvant chemotherapy which increases the survival but most of the patients are bare to the adverse effects of this therapy. Many studies have shown that paclitaxel and herceptin are effective for treating BCs. For this reason, the patient was administered neoadjuvant chemotherapy comprising these drugs. Metastasis did not occur in the 29-year-old woman. The effectiveness of preoperative neoadjuvent chemotherapy (paclitaxel and herceptin) was verified by surgery at a later point. When the patient underwent genetic testing, low levels of TYMS [10] and ERCC1 [11] expression were found, which indicated that chemotherapy with FOLFOX4 had been effective. No differences were found between the two pathological findings, indicating that the lesion treated with FOLFOX-4 was the same resistant clone of cancer cells or a new lesion. Although FOLFOX is effective, this type of chemotherapy is not mentioned in the NCCN guide. FOLFOX4 has previously been recommended as an adjuvant therapy for colon cancer. Adjuvant chemotherapy with FOLFOX works only in limited types of colon cancer (stage 2 vs. stage 3). Nevertheless, inclusion of FOLFOX4 in a treatment regimen can improve the survival of colorectal cancer patients. Ogata's study showed that FOLFOX4 is effective among patients with advanced colorectal cancer and that these patients can tolerate the treatment. Thus, the regimen shows great promise as second line therapy. A predictive model involving the genes listed herein can provide a highly accurate estimate of chemotherapy sensitivity to the FOLFOX4 regimen. FOLFOX4 is effective for pre-treated patients with BC. In any treatment plan, drug sensitivity rather than the disease should be considered [12]. Efficacy of the treatment improves when effective intervention measures are employed. The problem of treatment ineffectiveness should be addressed. A large number of cases will be required to illustrate the effectiveness of this method. Tumor specified here molecular description of the malignant tissue and individualization in the selection of proper agents. With pCR, patients can take advantage of the chemotherapy scheme for improved control and resection. Identifying biomarkers that can predict the treatment response can make individualized BC therapy feasible. The reply of BC patients to NAC [13] was predicted by chances of definite status of specific patient and varied characteristics were used to predict effectiveness of the treatment.

\section{Acknowledgement}

The authors would like to thank Dr. Hongmin $\mathrm{Li}$ for her insightful comments and suggestions.

\section{References}

1. Bear HD, Anderson S, Smith RE, Geyer CE, Mamounas EP, Fisher B, Paik S. Sequential preoperative or postoperative docetaxel added to preoperative doxorubicin plus cyclophosphamide for operable breast cancer: National Surgical Adjuvant Breast and Bowel Project Protocol B-27. J Clin Oncol 2006; 24: 2019-2027.

2. Stoetzer OJ, Fersching DM, Salat C, Steinkohl O, Gabka CJ, Hamann U, Nagel D. Circulating immunogenic cell death biomarkers HMGB1 and RAGE in breast cancer patients during neoadjuvant chemotherapy. Tumor Biol 2013; 34: 81-90.

3. van Hazel GA, Heinemann V, Sharma NK, Findlay MP, Ricke J, Peeters M, Rodríguez J. SIRFLOX: Randomized phase III trial comparing first-line mFOLFOX6 (plus or minus bevacizumab) versus mFOLFOX6 (plus or minus bevacizumab) plus selective internal radiation therapy in patients with metastatic colorectal cancer. J Clin Oncol 2016; 34: 1723-1731.

4. Luo HY, Li YH, Wang W, Wang ZQ, Yuan X, Ma D, Jia J. Single-agent capecitabine as maintenance therapy after induction of XELOX (or FOLFOX) in first-line treatment of metastatic colorectal cancer: randomized clinical trial of efficacy and safety. Annal Oncol 2016; 27: 1074-1081. 
5. Folprecht G, Pericay C, Saunders MP, Thomas A, Lopez Lopez R, Roh JK, Ackland SP. Oxaliplatin and 5-FU/folinic acid (modified FOLFOX6) with or without aflibercept in first-line treatment of patients with metastatic colorectal cancer: the AFFIRM study. Annal Oncol 2016; 27: 1273-1279.

6. Quidde J, Arnold D, Stein A. Clinical management of localized colon cancer with capecitabine. Clin Med Insights Oncol 2012; 6: 363.

7. Sharma G, Mirza S, Parshad R, Gupta SD, Ralhan R. DNA methylation of circulating DNA: a marker for monitoring efficacy of neoadjuvant chemotherapy in breast cancer patients. Tumor Biol 2012; 33: 1837-1843.

8. Croshaw R, Shapiro-Wright H, Svensson E, Erb K, Julian T. Accuracy of clinical examination, digital mammogram, ultrasound, and MRI in determining postneoadjuvant pathologic tumor response in operable breast cancer patients. Annal Surgical Oncology 2011; 18: 3160-3163.

9. Keam B, Im SA, Lim Y, Han SW, Moon HG, Oh DY, Kim DW. Clinical usefulness of AJCC response criteria for neoadjuvant chemotherapy in breast cancer. Annal Surgical Oncol 2013; 20: 2242-2249.

10. Langer R, Specht K, Becker K, Ewald P, Ott K, Lordick F, Höfler H. Comparison of Pretherapeutic and Postherapeutic Expression Levels of ChemotherapyAssociated Genes in Adenocarcinomas of the Esophagus
Treated by 5-Fluorouracil-and Cisplatin-Based Neoadjuvant Chemotherapy. Am J Clin Pathol 2007; 128: 191-197.

11. Smith DH, Fiehn AMK, Fogh L, Christensen IJ, Hansen TP, Stenvang J, Jensen SS. Measuring ERCC1 protein expression in cancer specimens: validation of a novel antibody. Scientific Reprt 2014.

12. Lu X, Pan J, Li S, Shen S, Chi P, Lin H, Huang S. Establishment of a predictive genetic model for estimating chemotherapy sensitivity of colorectal cancer with synchronous liver metastasis. Cancer Biother Radiopharmaceuticals 2013; 28: 552-558.

13. Takada M, Sugimoto M, Ohno S, Kuroi K, Sato N, Bando $\mathrm{H}$, Chow LWC. Predictions of the pathological response to neoadjuvant chemotherapy in patients with primary breast cancer using a data mining technique. Breast Cancer Res Treatment 2012; 134: 661-670.

\section{*Correspondence to}

Haifeng Cai

Department of Breast Surgery

Tangshan People's Hospital

PR China 